\title{
Measurement of the Cobb angle by 3D ultrasound: a valuable additional method for the prenatal evaluation of congenital scoliosis
}

\author{
Wenjie Mu, Miao He, Ting Lei, Lihe Zhang, Liu Du, Hongning Xie \\ Department of Ultrasonic in Obstetrics and Gynecology, the First Affiliated Hospital of Sun Yat-sen University, Guangzhou, China
}

Contributions: (I) Conception and design: H Xie, W Mu; (II) Administrative support: T Lei, L Du; (III) Provision of study materials or patients: L Zhang, M He; (IV) Collection and assembly of data: M He; (V) Data analysis and interpretation: W Mu; (VI) Manuscript writing: All authors; (VII) Final approval of manuscript: All authors.

Correspondence to: Hongning Xie. The First Affiliated Hospital of Sun Yat-Sen University, Zhongshan 2nd Road \#58, Guangzhou, Guangdong 510080, China. Email: xiehn@mail.sysu.edu.cn.

\begin{abstract}
Background: This study aimed to measure the Cobb angle of the fetal spine using three-dimensional ultrasound (3D-US) and to assess the relationship between the Cobb angle and the prognosis of congenital scoliosis.

Methods: From March 2015 to June 2019, 77 pregnant women whose fetuses had suspected spinal skeletal dysplasia consented to undergo 3D-US examinations, and 54 fetuses were selected for the analysis group. The study protocol was approved by the review board of the Institutional Ethics Committee for Fetal Medicine. 3D-US was used to show the structure of the fetal spine in 3 planes, and the Cobb angle was measured on the coronal plane. The diagnostic efficacy of 3D-US was compared to that of X-ray for 33 fetuses.

Results: In the diagnosis of congenital scoliosis, the sensitivity, specificity, accuracy, positive predictive value (PPV), and negative predictive value (NPV) of 3D-US were 91.7\%, 90.0\%, 90.7\%, 88.0\%, and 93.1\%, respectively. The area under the receiver operating characteristic (ROC) curve with 3D-US was 0.908. The Spearman correlation coefficient between the Cobb angle measurement on an X-ray image and on the coronal plane image acquired by $3 \mathrm{D}-\mathrm{US}$ was 0.84 , which showed a significant correlation $(\mathrm{P}<0.05)$.

Conclusions: 3D-US was successful in the diagnosis of congenital scoliosis. It is feasible to measure the Cobb angle on the coronal plane of the fetal spine by using 3D-US. The Cobb angle has the potential to become an auxiliary index for evaluating the prognosis of congenital scoliosis.
\end{abstract}

Keywords: Congenital scoliosis; 3D/4D ultrasound; obstetric ultrasound; Cobb angle; skeletal dysplasia

Submitted May 25, 2021. Accepted for publication Dec 29, 2021; Published: 02 Mar 2022.

doi: $10.21037 /$ qims-21-558

View this article at: https://dx.doi.org/10.21037/qims-21-558

\section{Introduction}

Caused by complicated interactions between genetic and environmental factors, congenital scoliosis includes a heterogeneous and complex group of skeletal dysplasias (1). Segmental and developmental disorders of the vertebral body in the embryonic stage result in serious congenital spinal deformities. These conditions are often complicated with neural tube dysplasia and other malformations (2). Because of the asymmetric growth and development of both sides of the spine, the deformity is progressively aggravated with increasing age. Two recent reports demonstrated a significantly higher incidence of intraspinal anomalies in 
patients with congenital scoliosis and rib abnormalities than in those without $(3,4)$.

Routine two-dimensional ultrasound (2D-US) is the main prenatal examination method for screening skeletal disorders. It is used to identify abnormalities in fetal bones, primarily osteogenesis imperfecta. However, due to the complexity and diversity of these skeletal disorders, the accuracy of prenatal diagnosis of these diseases by 2D-US remains approximately $65 \%$ in Britain $(5,6)$. Previous studies have suggested that a three-dimensional ultrasound (3D-US) may have better sensitivity than 2D-US for the antenatal diagnosis and characterization of skeletal dysplasias $(7,8)$. The application of 3D-US seems to show a more accurate prenatal diagnosis of congenital scoliosis by obtaining coronal plane images of the fetal spine. However, except for cases with other serious complications or genetic defects, there are no semi-quantitative or quantitative diagnostic criteria for congenital scoliosis found in prenatal screening. Therefore, the relationship between fetal scoliosis severity and prognosis requires further evaluation and observational research. The prognosis of congenital scoliosis varies, however, prenatal diagnosis of the severity of congenital scoliosis is lack of quantitative indicators. In patients with adolescent idiopathic scoliosis, the Cobb angle has been shown to be a reliable method for assessing curvature severity using $\mathrm{X}$-ray screening $(9,10)$. However, there is currently no literature that has assessed whether the Cobb angle could be measured on fetal spine coronal images to provide information for the prognosis of congenital scoliosis.

This study measured the Cobb angle on coronal plane images of the fetal spine using 3D-US, with the final diagnosis made by postmortem or postnatal radiological examination. In addition, by following up the children born with congenital scoliosis, we assessed the relationship between the Cobb angle and the prognosis of the disease.

We present the following article in accordance with the standards for reporting of diagnostic accuracy studies (STARD) reporting checklist (available at https://qims. amegroups.com/article/view/10.21037/qims-21-558/rc).

\section{Methods}

\section{General design}

From March 2015 to June 2019, this retrospective study recruited women with singleton pregnancies of 18-38 weeks who underwent special ultrasound examinations of the fetal spine in the Department of Ultrasonic in Obstetrics and
Gynecology of the First Affiliated Hospital of Sun YatSen University. Fetuses with spinal skeletal dysplasia were suitable for inclusion in our study. Cases were excluded if they satisfied the following criteria: (I) structural malformations other than fetal spinal skeletal dysplasia were found during the examination; (II) fetal macrosomia or intrauterine growth restriction were observed; (III) the pregnant women had clinical symptoms that could affect fetal growth (such as diabetes, chronic hypertension, and preeclampsia); or (IV) the image quality of fetal spine was too poor to show vertebral alignment. The study protocol was approved by the review board of Institutional Ethics Committee for Fetal Medicine. As we were using a standard of care clinical protocol, the responsible ethics committee did not require additional approval for the non-interventional design of our retrospective study. All the women gave informed consent to participate in the study. The study was conducted in accordance with the Declaration of Helsinki (as revised in 2013).

\section{Patients}

A total of 77 pregnant women whose fetuses had suspected fetal spinal skeletal dysplasia and who consented to undergo a $3 \mathrm{D}-\mathrm{US}$ examination following a conventional 2D-US examination were included in this study. Their ages ranged from 19 to 45 years, with a mean age of 29.3 years. The gestational ages (GAs) of the fetuses were $24.9 \pm 3.1$ weeks. Of the 77 cases, 18 were failed to be followed up because the imaging data were incomplete or no further examination was performed after induced labor. Pregnancy outcome confirmation and follow-up of Child's development were performed in 59 cases.

\section{D-US examination and measurement of the Cobb angle}

A conventional 2D-US and a $3 \mathrm{D}$-US examination were performed by a radiologist with over 30 years of experience in prenatal ultrasound diagnosis. To test the consistency of the obtained measurements, another radiologist with 9 years of experience also performed the same examinations back-to-back, based on the stored images. Two ultrasound scanners were used: an RAB6-D and an eM6C G2 (GE Healthcare, Milwaukee, WI, USA). The probe power was less than $100 \mathrm{~mW} / \mathrm{cm}$.

After a traditional ultrasound examination of the fetal structure, the 3D skeletal mode setting was used to obtain images of the fetal spine in 3 directions. To acquire the 

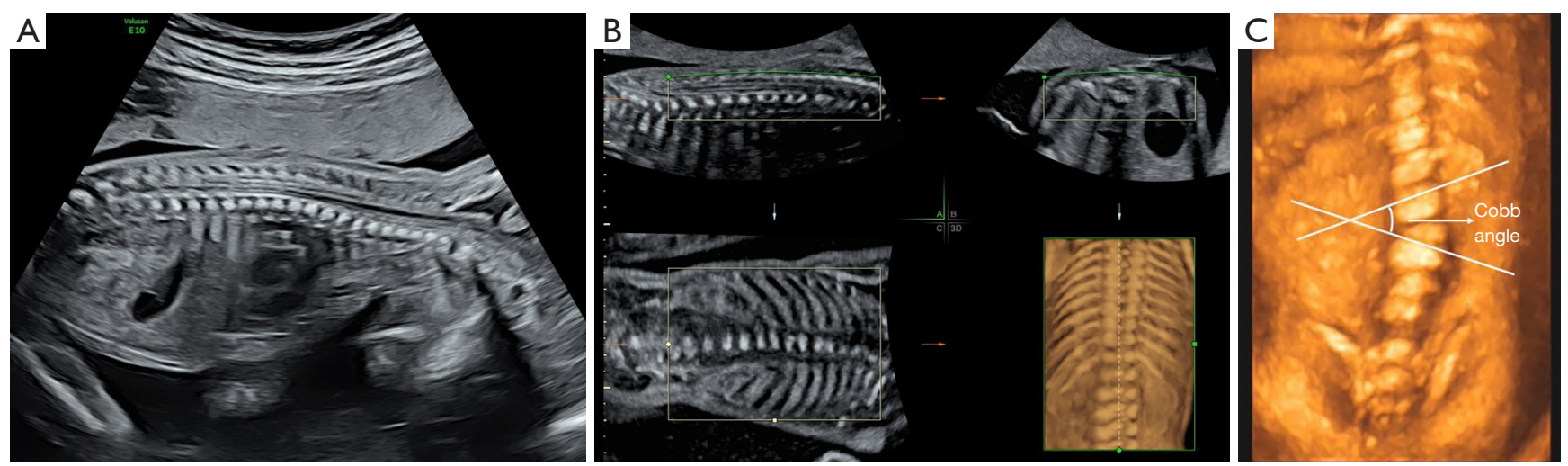

Figure 1 The process of obtaining coronal images of the fetal spine by three-dimensional ultrasound (3D-US) and the measurement of the Cobb angle. (A) A normal case at a gestational age of 26 weeks. The whole sagittal plane of the fetal spine should be displayed as much as possible in the ultrasonography scanning process. Then, the preset 3D mode of skeleton imaging should be selected, with the region of interest set to include the spine. (B) After obtaining the 3D data, adjustments to the 3 planes should be made to represent the fetal spinal sagittal plane (upper right, plane A), the longitudinal plane (upper left, plane B), and coronal image (lower right, plane 3D). Plane 3D displays a threedimensional image of the coronal plane of the fetal spine, which can be enlarged and adjusted to make the long axis of the spine vertical. (C) The 3D-US display of the coronal plane of a 23-week-old fetal spine diagnosed with hemivertebra (the third lumbar vertebra). Scoliosis is defined as a lateral deviation of the spine. The Cobb angle should be measured at the scoliosis segment, with lines drawn across the vertebral column on the upper surface of the upper vertebra and the lower surface of the lower vertebra. The angle measured may be either that between these 2 lines or that between lines drawn perpendicular to them. In this case the measured Cobb angle was $32.7^{\circ}$.

entire spine, the volume box and the angle for volume sampling were varied depending on GA and the bone involved (Figure 1A,1B). These images were stored on the hard drive of the machine and analyzed. Scoliosis was defined as a lateral deviation of the spine. On the coronal plane images of the fetal spine acquired by $3 \mathrm{D}-\mathrm{US}$, the Cobb angle measurement was based on the 2 most tilted cranial and caudal vertebrae on the radiographs, seen from the apex of the curve of scoliosis and defined as the "for a given group of vertebrae, lines are drawn across the vertebral column on the upper surface of the upper vertebra and the lower surface of the lower vertebra". The angle measured may be either that between these 2 lines or that between lines drawn perpendicular to them (Figure 1C).

The Cobb angles measured by $3 \mathrm{D}$-US imaging were compared with radiological images on postnatal or postmortem (following induced labor) examination. Microsoft Office Excel 2003 (Microsoft Corporation, Redmond, Washington) was used for data collection. Statistical analysis was performed using SPSS version 13 software (SSPS Inc., Chicago, IL, USA) and MedCalc (MedCalc Software, Mariakerke, Belgium). Receiver operating characteristic (ROC) curves were used to describe the diagnostic performance of the 3D-US. The
Spearman correlation coefficient was calculated to assess the relationship between the Cobb angle measurements on $\mathrm{x}$-ray examination and on the coronal plane of the fetal spine image acquired by $3 \mathrm{D}-\mathrm{US}$. The difference was considered significant when $\mathrm{P}<0.05$.

The reliability of the Cobb angles measured by $3 \mathrm{D}-$ US image was evaluated by a 2-way mixed model singlemeasurement intraclass correlation coefficient (ICC) for absolute agreement. Intra-observer reliability was evaluated by comparing the measurements obtained by each observer at different times (1 week apart) with a different order of measurements and calculating intra-observer ICCs for observer A and observer B. Interobserver reliability was evaluated by comparing the independent measurements obtained by the 2 observers. All ICCs were calculated with $95 \%$ confidence interval (CI), and the reliability was defined as slight (0-0.2), fair (0.21-0.4), moderate (0.41-0.6), substantial $(0.61-0.8)$, or almost perfect $(0.81-1.0)$ for clinical application. Absolute agreement was determined by analyzing intra- and interobserver variations for categorical variables using an extension of the Bland-Altman method for continuous variables, whereby the $95 \%$ CI of the agreement was calculated. If the lower limit of the $95 \%$ CI was less than 0.50 , the agreement was considered poor. 


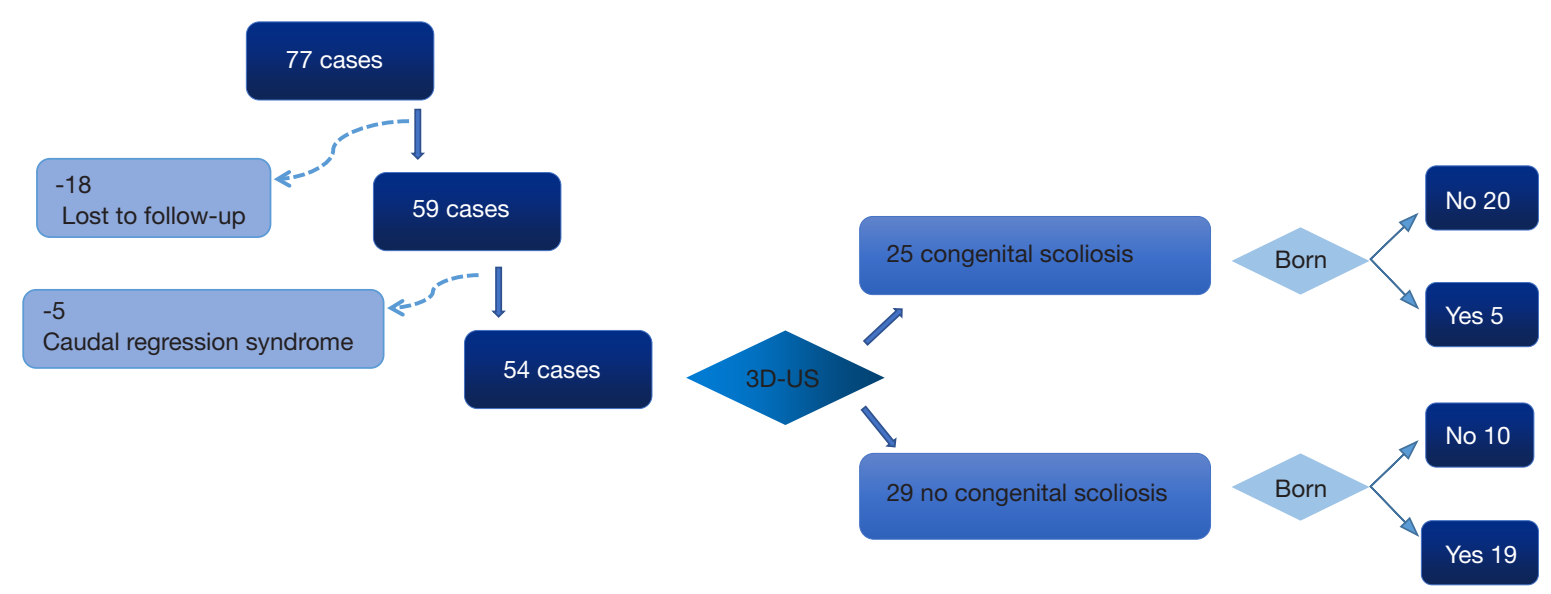

Figure 2 A flow chart showing participant enrolment and analysis.

Table 1 Anatomic abnormalities in 54 patients with or without congenital scoliosis diagnosis by $3 \mathrm{D}$-US

\begin{tabular}{ll}
\hline Diagnosis & $\mathrm{n}$ \\
\hline Congenital scoliosis & 25 \\
Hemivertebra & 14 \\
Multiple vertebral dysplasia & 5 \\
Fused vertebrae & 3 \\
Calcification dysplasia & 2 \\
Vertebral dysplasia & 1 \\
No congenital scoliosis & 29 \\
Calcification dysplasia & 14 \\
Multiple vertebral dysplasia & 6 \\
Hemivertebra & 4 \\
Trigonometric vertebra & 2 \\
Vertebral dysplasia & 1 \\
Butterfly vertebrae & 1 \\
Hemivertebrae + Fused vertebrae & 1 \\
\hline
\end{tabular}

3D-US, three-dimensional ultrasound.

\section{Results}

Of the 77 fetuses, 18 were loss of follow-up, and 5 were diagnosed with caudal regression syndrome, which did not cause congenital scoliosis. These 23 fetuses were excluded from the final analysis group. Of the 54 remaining fetuses, 29 were found not to have congenital scoliosis by $3 \mathrm{D}$-US. Of these, 19 were born, and the remaining 10 pregnancies were terminated. Of the 25 fetuses diagnosed with congenital scoliosis by $3 \mathrm{D}-\mathrm{US}, 5$ cases were born, and the remaining 20 pregnancies were terminated (Figure 2).

The most common anatomic abnormality in the fetuses with congenital scoliosis was hemivertebra(one side of a vertebra fails to develop completely). For those without congenital scoliosis, the most common anatomic abnormality was calcification dysplasia (uneven calcification of vertebral body) (Table 1). The number and location of the vertebral abnormalities are shown in Figure 3. In addition, 25 of 54 cases chose to use prenatal chromosome and gene detection, and 5 of 25 cases revealed abnormal findings.

The sensitivity, specificity, accuracy, positive predictive value (PPV), and negative predictive value (NPV) of the 3D-US were $91.7 \%, 90.0 \%, 90.7 \%, 88.0 \%$, and $93.1 \%$, respectively. The area under the ROC curve for $3 \mathrm{D}$ US was 0.908 (Figure 4). The Spearman correlation coefficient between the Cobb angle measurement on $\mathrm{X}$-ray examination and on the coronal plane of the fetal spine image acquired by $3 \mathrm{D}$-US was 0.84 , which showed a significant correlation $(\mathrm{P}<0.05)$. The interobserver ICC of the Cobb angle measurements was 0.890 (95\% CI: 0.882-0.907), whereas the intra-observer ICC for observer A was 0.852 (95\% CI: 0.889-0.917), and for observer B was 0.828 (95\% CI: 0.889-0.917). Absolute agreement values between the 3D-US-based and the X-ray-based Cobb angle measurements were calculated, and the ICCs were 0.876 (95\% CI: 0.832-0.912) for observer A and 0.857 (95\% CI: $0.830-0.872$ ) for observer B.

Among the 29 fetuses diagnosed were found not to have congenital scoliosis by 3D-US, 19 were born, and the followup range was from 1 to 42 months. Spinal appearance, 

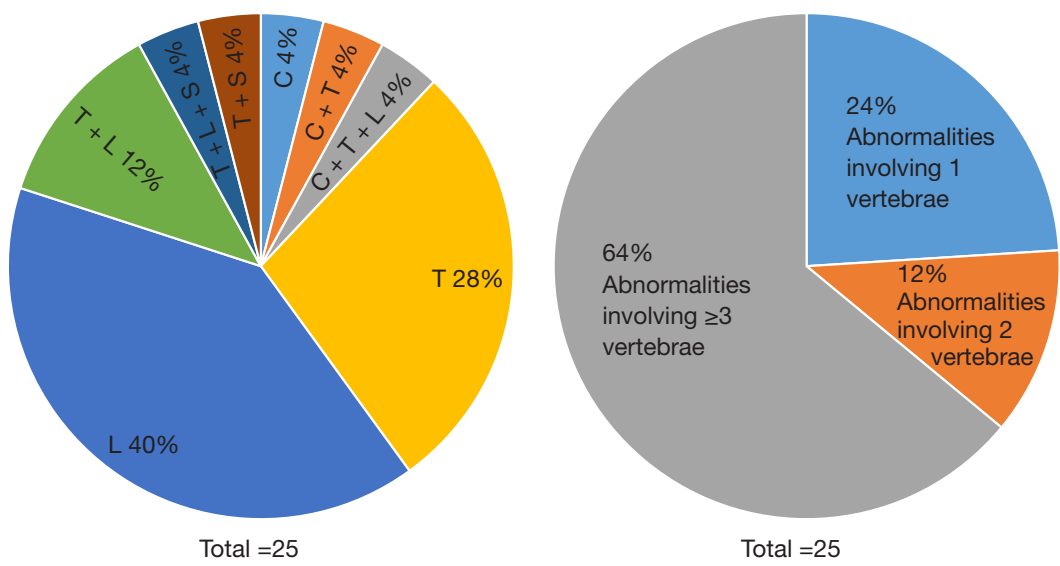

Figure 3 A pie chart showing the abnormal vertebral body distribution. C: cervical vertebra; T: thoracic vertebra; L: lumbar vertebra; S: sacral vertebra.

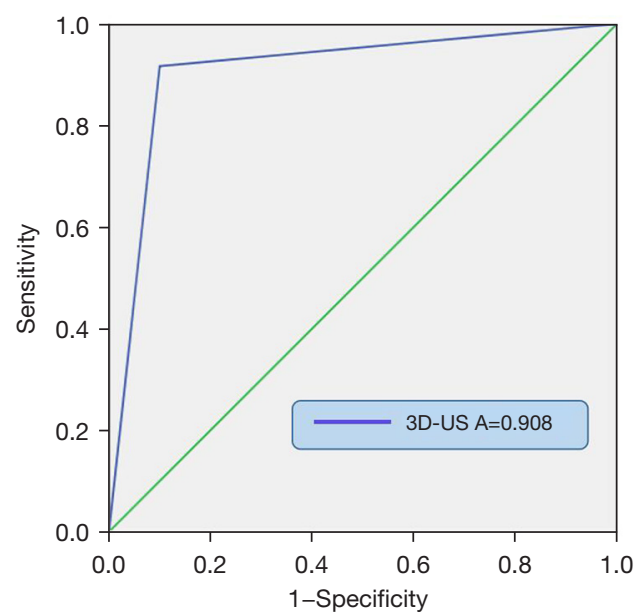

Figure 4 Receiver operating characteristic (ROC) curves for 3-D ultrasound (3D-US) diagnosis of fetal scoliosis. walking ability, and athletic performance in 18 of the babies were not significantly affected at the final follow-up. Only 1 baby was diagnosed with closed spina bifida after birth and underwent surgery at 5 months. At the last follow-up, this baby was 1 year and 9 months old, and his walking ability and athletic performance lagged behind those of his peers. Among the 25 fetuses diagnosed with congenital scoliosis by $3 \mathrm{D}-\mathrm{US}, 5$ were born. The Cobb angles measured on the coronal plane images of their fetal spines were $45.8^{\circ}, 32.7^{\circ}$, $17.9^{\circ}, 24.2^{\circ}$, and $22.9^{\circ}$. Prenatal imaging data, together with postnatal findings, are shown in Table 2.

\section{Discussion}

A prenatal diagnosis of skeletal dysplasia is often difficult to make, especially in the absence of family history. Most

Table 2 Prenatal diagnosis and postnatal findings in the group of patients with congenital scoliosis diagnosed by 3D-US

\begin{tabular}{|c|c|c|c|c|c|c|c|c|}
\hline Case & $\begin{array}{c}\text { Gestational age } \\
\text { at diagnosis } \\
\text { (weeks) }\end{array}$ & Diagnosis & \multicolumn{2}{|c|}{ Cobb angle } & \multicolumn{4}{|c|}{ Follow-up result } \\
\hline 2 & 25 & Calcification dysplasia in T7-L9 & $32.7^{\circ}$ & $34.3^{\circ}$ & 44 & Slightly convex to the left & Normal & Normal \\
\hline 4 & 24 & Hemivertebra in L2 & $24.2^{\circ}$ & $22.5^{\circ}$ & 18 & Normal & Normal & $\begin{array}{l}\text { Slightly slower } \\
\text { than peers }\end{array}$ \\
\hline 5 & 24 & Hemivertebrae in T8, T9 & $22.9^{\circ}$ & $21.4^{\circ}$ & 20 & Normal & Normal & Normal \\
\hline
\end{tabular}

3D-US, three-dimensional ultrasound. 
skeletal system abnormalities are caused by autosomal dominant or autosomal recessive inheritance, either from a new gene mutation or from typical family history. Even if there is a family history of dominant inheritance, the clinical performance of each patient is different and depends on the degree of gene expression $(2,11)$. In our study, 25 fetuses with spinal abnormalities were examined for chromosomal variations, 6 variations were identified, 5 of which were considered benign heteromorphoses. In diagnosing fetal spinal abnormalities, analysis of Karyotyping of chromosome by amniocentesis may not be able to provide enough information and more specific examinations and prognosticrelated analyses are also necessary. Unfortunately, there is little literature on the relationship between the prenatal ultrasound evaluation of congenital scoliosis and prognostic features.

We found that hemivertebra was the most common anatomical anomaly leading to scoliosis. Hemivertebra is defined as a wedge-shaped vertebra with 1 pedicle on 1 side. Hemivertebra can be subdivided into fully segmented, semi-segmented, and unsegmented variants based on the relationship with the cranial and caudal adjacent vertebral bodies. McMaster (12) reported that the variant most at risk for progression was a unilateral bar with contralateral hemivertebra, followed by a unilateral bar, hemivertebra, wedge vertebra, and block vertebra, the last of which is the least likely to cause any significant deformity. We also found that multiple vertebral abnormalities are more likely to lead to scoliosis, and the most common site of deformity is the lumbar spine, followed by the thoracic spine. McMaster also reported the rate of deterioration in patients with various types of congenital spine anomalies (13). Unfortunately, this was a postnatal observational study with no information on prenatal diagnosis. Determining which congenital curves will progress rapidly is a difficult proposition, making additional observations and research related to prognosis necessary. Pre-delivery diagnosis and prognostic evaluation would greatly benefit a patient's family.

$2 \mathrm{D}$-US is the method routinely used for prenatal detection of most abnormalities. It has a sensitivity of approximately $60 \%$ and the $3 \mathrm{D}$-US method has been reported to have better diagnostic efficiency for various fetal abnormalities $(5,6)$, including skeletal system abnormalities such as facial dysmorphisms and anomalies involving the limbs. Because of its physical characteristics, 2D-US has obvious deficits in the display of the fetal spine in the coronal plane, the most crucial view for diagnosing scoliosis. The 3D-US can display the coronal plane, sagittal plane, and longitudinal plane of the fetal spine clearly.
By adjusting the view of the coronal plane, the structural arrangement of the vertebral body can be clearly seen. The ability to observe whether the spine is vertical and/ or whether there is left or right bending in $3 \mathrm{D}-\mathrm{US}$ is also adequate. Consequently, the diagnostic efficiency of two dimensional ultrasound for fetal spinal deformity is greatly improved. In this study, 3D-US showed high sensitivity and specificity for evaluating congenital scoliosis.

Most skeletal system malformations can be screened by prenatal ultrasound examination. However, it is often difficult to make a specific diagnosis. A final diagnosis of skeletal system malformations often depends on postnatal radiology. As observed in the present study, most skeletal dysplasia cases were detected late in the second or third trimester of pregnancy. In this study, the diagnostic efficiencies of 3D-US and X-ray examinations were highly correlated. Furthermore, as a non-radioactive examination, 3D-US could promote safer, more accurate diagnosis and aid in clinical decision-making in obstetrics. In clinical work, we observed that many fetuses with congenital scoliosis but no other structural or chromosomal abnormalities achieved satisfactory prognosis and postnatal development. Therefore, a parameter that could correlate the degree of fetal spinal curvature with prognosis is necessary. As the spine develops, and weight bearing changes occur in the growing child, other, more complex evaluative systems for spinal surgery can be employed, such as the Lenke method $(14,15)$. As a preliminary study, we chose a relatively simple index - the Cobb angle - to analyze the fetal spine in the coronal plane on 3D-US, which, to our knowledge, has not been reported in previous studies. Measurement of the Cobb angle is the standard technique for evaluating radiographs for spinal deformities and is used to document curve progression, select the method of treatment, and evaluate treatment outcomes (16-18). This study measured the Cobb angle in all 25 fetuses with scoliosis confirmed by ultrasound and 5 fetuses were born. The Cobb angles measured on the coronal plane images of those 5 fetal spines were $45.8^{\circ}$, $32.7^{\circ}, 17.9^{\circ}, 24.2^{\circ}$, and $22.9^{\circ}$ (Figure 5 ). All 5 babies were followed up by regular visits until they learned to walk. Only 1 baby, whose Cobb angle was $45.8^{\circ}$, showed athletic ability behind that of his peers. The paediatric spinal surgeon advised surgery and long-term follow-up for this patient. For Cobb angles measured on radiographs, a Cobb angle greater than $40^{\circ}$ typically indicates that spinal surgery is needed to correct the deformity (18), consistent with our observations in this study. However, whether 40 degrees is suitable as the diagnostic threshold for ultrasonic measurement 


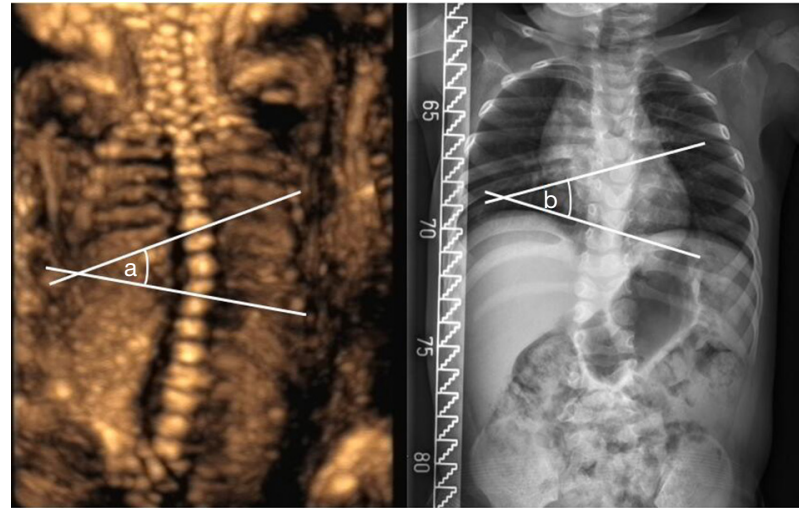

Figure 5 (A) Three-dimensional ultrasound (3D-US) display of the coronal plane of a 25 -week-old fetal spine, on which the Cobb angle (a) was measured at $32.7^{\circ}$. (B) Three years and 8 months after the birth of the same fetus, the Cobb angle (b) measured on an $\mathrm{X}$-ray image was $34.3^{\circ}$.

requires further study. In 2018, a study of 101 children with adolescent idiopathic scoliosis suggested that the Cobb angle could be measured reliably on US images with specific algorithms (19). We believe that the measurement of the fetal Cobb angle is more meaningful because it provides an earlier evaluation index for clinical decision-making. Termination of the pregnancy was recommended in cases where congenital scoliosis was combined with other serious deformities (3). Chromosomal abnormality testing is needed for fetuses with simple scoliosis, while fetuses with negative test results could be kept under continuous observation and follow-up. In these cases, the Cobb angle becomes an important evaluation index.

Our study had several limitations. First, the manipulation of $3 \mathrm{D}-\mathrm{US}$ is somewhat experience-dependent. Correctly acquiring $3 \mathrm{D}$ images of the fetal spine requires practice. Second, the sample size for this study was small, especially the number of fetuses born with congenital scoliosis, which made statistical analysis difficult. Whether these measurements can provide parameters for the quantitative analysis of prognoses still requires a large-scale, multicenter study. Third, the feasibility and image quality of 3DUS may decrease as gestation progresses. Of the 54 patients in this study, 17 were of a GA of between 18 weeks and 23 weeks +6 days, 28 were of a GA of between 24 weeks and 27 weeks+ 6 days, and 9 cases were of a GA between 28 weeks and 33 weeks +5 days. The sensitivity of $3 \mathrm{D}-\mathrm{US}$ in the diagnosis of congenital scoliosis in each GA group were $88.2 \%, 92.9 \%$, and $88.9 \%$, respectively. The difference was not statistically significant. We suggest that pregnant women whose fetuses have suspected spinal deformity should be consulted by a prenatal diagnostic consultation center as soon as possible. We also believe that multiple examinations at different GAs for each patient are necessary to observe the process of change and provide more information to guide prognosis.

\section{Conclusions}

The method of 3D-US can clearly show 3D anatomical characteristics of the fetal spinal structure, vividly express the curvature of the spine, and directly display the shape of each vertebral body. In our study, 3D-US successfully diagnosed congenital scoliosis. Its diagnostic efficiency was also highly correlated with that of radiological detection. We suggest that the prenatal Cobb angle be measured on the coronal plane image of the fetal spine obtained from 3D-US and used as an auxiliary index for the prognostic evaluation of a fetus with congenital scoliosis.

\section{Acknowledgments}

As the first author, WM would very much like to thank her friend, Jiale Wei, from Guangzhou Sports University. His understanding, support, and encouragement have been a source of light always with her throughout many days and nights of writing.

Funding: This study was supported by research grants from the National Scientific Foundation Committee of China (No. 82171938 and No. 81801705).

\section{Footnote}

Reporting Checklist: The authors have completed the standards for reporting of diagnostic accuracy studies (STARD) reporting checklist. Available at https://qims. amegroups.com/article/view/10.21037/qims-21-558/rc

Conflicts of Interest: All authors have completed the ICMJE uniform disclosure form (available at https://qims. amegroups.com/article/view/10.21037/qims-21-558/coif). The authors have no conflicts of interest to declare.

Ethical Statement: The authors are accountable for all aspects of the work in ensuring that questions related to the accuracy or integrity of any part of the work are appropriately investigated and resolved. The study was conducted in 
accordance with the Declaration of Helsinki (as revised in 2013). The study was approved by the Institutional Ethics Committee for Fetal Medicine, and individual consent for this retrospective analysis was obtained.

Open Access Statement: This is an Open Access article distributed in accordance with the Creative Commons Attribution-NonCommercial-NoDerivs 4.0 International License (CC BY-NC-ND 4.0), which permits the noncommercial replication and distribution of the article with the strict proviso that no changes or edits are made and the original work is properly cited (including links to both the formal publication through the relevant DOI and the license). See: https://creativecommons.org/licenses/by-nc-nd/4.0/.

\section{References}

1. Dugoff L, Thieme G, Hobbins JC. Skeletal anomalies. Clin Perinatol 2000;27:979-1005.

2. Bulman MP, Kusumi K, Frayling TM, McKeown C, Garrett C, Lander ES, Krumlauf R, Hattersley AT, Ellard S, Turnpenny PD. Mutations in the human delta homologue, DLL3, cause axial skeletal defects in spondylocostal dysostosis. Nat Genet 2000;24:438-41.

3. Ghandhari H, Tari HV, Ameri E, Safari MB, Fouladi DF. Vertebral, rib, and intraspinal anomalies in congenital scoliosis: a study on 202 Caucasians. Eur Spine J 2015;24:1510-21.

4. Shen J, Wang Z, Liu J, Xue X, Qiu G. Abnormalities associated with congenital scoliosis: a retrospective study of 226 Chinese surgical cases. Spine (Phila Pa 1976) 2013;38:814-8.

5. Doray B, Favre R, Viville B, Langer B, Dreyfus M, Stoll C. Prenatal sonographic diagnosis of skeletal dysplasias. A report of 47 cases. Ann Genet 2000;43:163-9.

6. Garjian KV, Pretorius DH, Budorick NE, Cantrell CJ, Johnson DD, Nelson TR. Fetal skeletal dysplasia: three-dimensional US--initial experience. Radiology 2000;214:717-23.

7. Krakow D, Williams J 3rd, Poehl M, Rimoin DL, Platt LD. Use of three-dimensional ultrasound imaging in the diagnosis of prenatal-onset skeletal dysplasias. Ultrasound Obstet Gynecol 2003;21:467-72.

8. Ruano R, Molho M, Roume J, Ville Y. Prenatal diagnosis of fetal skeletal dysplasias by combining two-dimensional and three-dimensional ultrasound and intrauterine threedimensional helical computer tomography. Ultrasound Obstet Gynecol 2004;24:134-40.

9. Facanha-Filho FA, Winter RB, Lonstein JE, Koop S,
Novacheck T, L'Heureux EA Jr, Noren CA. Measurement accuracy in congenital scoliosis. J Bone Joint Surg Am 2001;83:42-5.

10. Loder RT, Urquhart A, Steen H, Graziano G, Hensinger RN, Schlesinger A, Schork MA, Shyr Y. Variability in Cobb angle measurements in children with congenital scoliosis. J Bone Joint Surg Br 1995;77:768-70.

11. Basu PS, Elsebaie H, Noordeen MH. Congenital spinal deformity: a comprehensive assessment at presentation. Spine (Phila Pa 1976) 2002;27:2255-9.

12. McMaster MJ, David CV. Hemivertebra as a cause of scoliosis. A study of 104 patients. J Bone Joint Surg Br 1986;68:588-95.

13. McMaster MJ, Ohtsuka K. The natural history of congenital scoliosis. A study of two hundred and fifty-one patients. J Bone Joint Surg Am 1982;64:1128-47.

14. Smith JS, Shaffrey CI, Bess S, Shamji MF, Brodke D, Lenke LG, Fehlings MG, Lafage V, Schwab F, Vaccaro AR, Ames CP. Recent and Emerging Advances in Spinal Deformity. Neurosurgery 2017;80:S70-85.

15. Puno RM, An KC, Puno RL, Jacob A, Chung SS. Treatment recommendations for idiopathic scoliosis: an assessment of the Lenke classification. Spine (Phila Pa 1976) 2003;28:2102-14; discussion 2114-5.

16. Shea KG, Stevens PM, Nelson M, Smith JT, Masters KS, Yandow S. A comparison of manual versus computerassisted radiographic measurement. Intraobserver measurement variability for Cobb angles. Spine (Phila Pa 1976) $1998 ; 23: 551-5$.

17. Morrissy RT, Goldsmith GS, Hall EC, Kehl D, Cowie GH. Measurement of the Cobb angle on radiographs of patients who have scoliosis. Evaluation of intrinsic error. J Bone Joint Surg Am 1990;72:320-7.

18. Negrini S, Donzelli S, Aulisa AG, Czaprowski D, Schreiber S, de Mauroy JC, et al. 2016 SOSORT guidelines: orthopaedic and rehabilitation treatment of idiopathic scoliosis during growth. Scoliosis Spinal Disord 2018;13:3.

19. Brink RC, Wijdicks SPJ, Tromp IN, Schlösser TPC, Kruyt MC, Beek FJA, Castelein RM. A reliability and validity study for different coronal angles using ultrasound imaging in adolescent idiopathic scoliosis. Spine J 2018;18:979-85.

Cite this article as: $\mathrm{Mu} \mathrm{W}, \mathrm{He} M$, Lei T, Zhang L, Du L, Xie $\mathrm{H}$. Measurement of the Cobb angle by $3 \mathrm{D}$ ultrasound: a valuable additional method for the prenatal evaluation of congenital scoliosis. Quant Imaging Med Surg 2022;12(5):28052812. doi: 10.21037/qims-21-558 